\title{
Multiple coronary artery-left ventricular fistulae: haemodynamic quantification by intracoronary Doppler ultrasound
}

\author{
Achim Meissner, Markus Lins, Gunhild Herrmann, Rüdiger Simon
}

Department of Cardiology, $1^{\text {st }}$ Medical Clinic, University of Kiel, Schittenhelmstrasse 12, D-24105 Kiel, Germany

Correspondence to: Dr Meissner.

Accepted for publication 24 March 1997

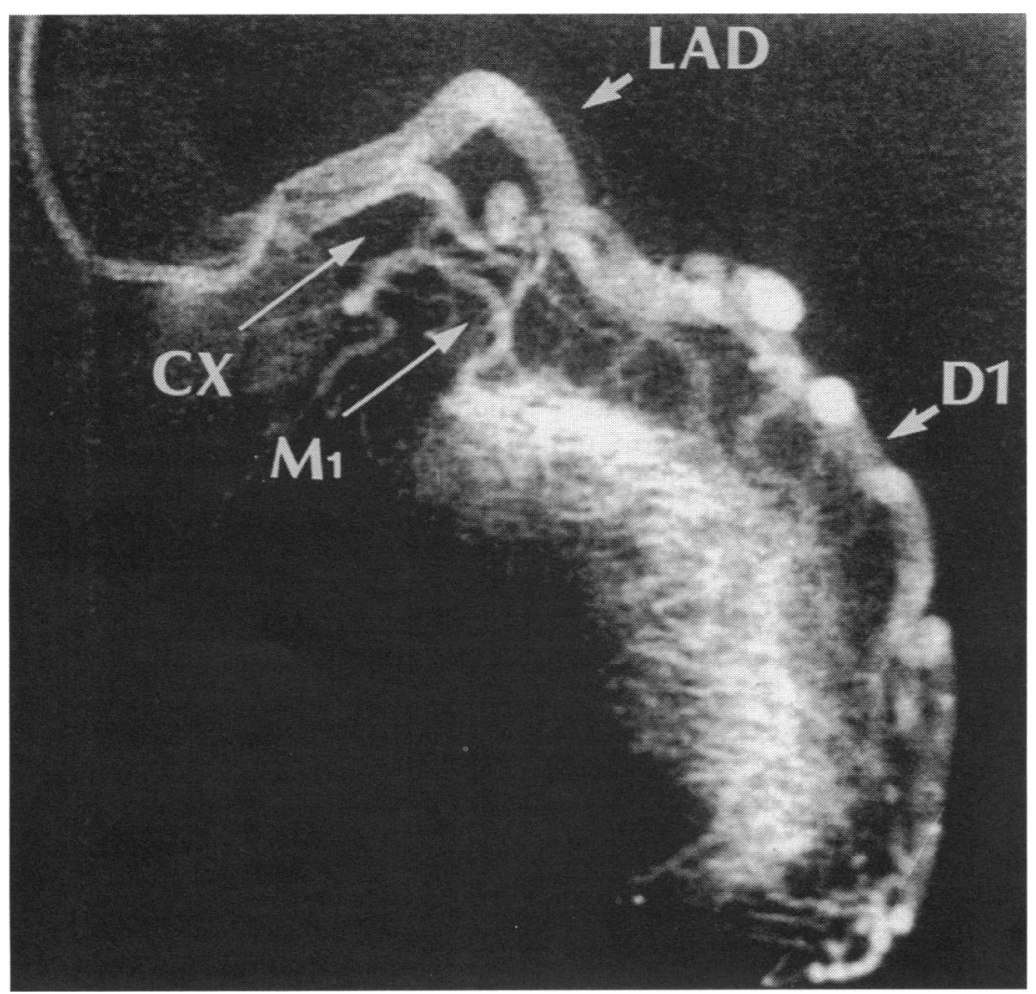

Figure 1 Selective coronary arteriography $\left(30^{\circ}\right.$ right anterior oblique view) showing the left anterior descending (LAD) and circumflex (CX) arteries. Multiple fine fistulae arising from the first diagonal branch (D1) and the first marginal branch (M1) communicate with the left ventricular cavity.

(Heart 1997;78:91-93)

Keywords: coronary fistulae; intracoronary ultrasound; Keywords: coronary
coronary anomaly

\begin{abstract}
Multiple coronary artery-left ventricular fistulae involving all three major coronary arteries are extremely rare. Clinical findings are heterogeneous but include a history of typical or atypical angina pectoris in most cases. Coronary arteriography in a 65 year old woman who presented with chest pain at rest revealed multiple fine fistulae arising from the left anterior descending, left circumflex, and right coronary arteries. Left-to-left shunt was estimated by measurements of coronary artery flow velocity with intravascular Doppler ultrasound.
\end{abstract} gram showed sinus rhythm ( 65 beats $/ \mathrm{min}$ ) with minor $(0.1 \mathrm{mV})$ ST segment elevations in leads II, III, and aVF. This appeared suggestive of acute myocardial infarction and prompted thrombolytic treatment with intravenous recombinant tissue plasminogen activator (rtPA). In addition, the patient received intravenous heparin, aspirin, and nitrates. Serum enzyme activities of creatinine kinase (CK) and CK-MB reached a maximum of 266 and $35 \mathrm{U} / 1$, respectively. Chest $x$ ray appeared normal. The clinical course was uneventful and the patient was transferred to the ward after two days.

In the bicycle exercise test a submaximal workload (150 W) was achieved after one week. No clinical or electrocardiographical signs of myocardial ischaemia were inducible. Heart catheterisation and coronary angiography were performed on day 14 . Left ventricular angiography revealed a normal sized hyper- 


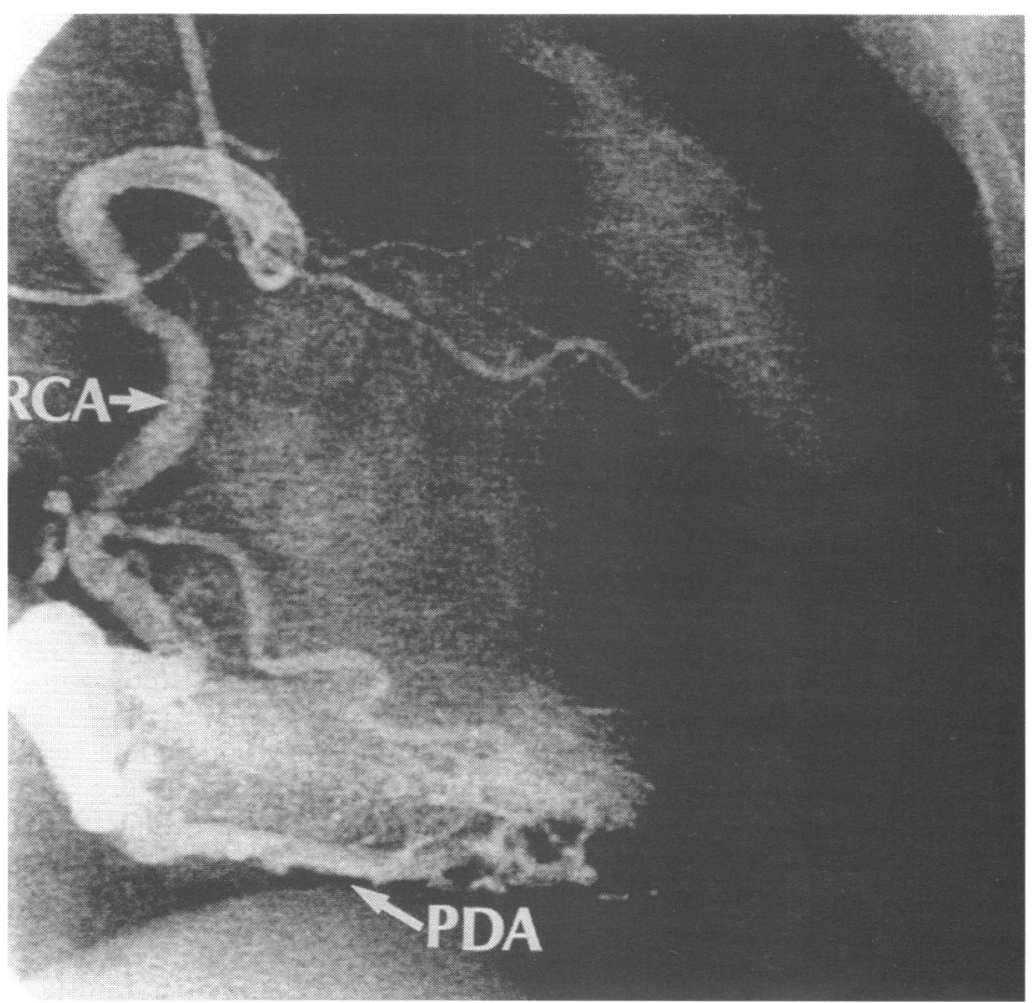

Figure 2 Selective coronary arteriography ( $30^{\circ}$ right anterior oblique view) showing the right coronary artery (RCA). A maze of fine vessels arise from the posterior descending artery $(P D A)$ and communicate with the left ventricular cavity.

trophic left ventricle (end diastolic volume index $76 \mathrm{ml} / \mathrm{m}^{2}$ ) without regional wall motion abnormalities, and an ejection fraction of $69 \%$. Selective arteriography of the left coronary artery was perturbed by the precipitous dilution of the contrast medium resulting from accelerated flow. The proximal part of the left anterior descending artery (LAD), the first diagonal branch, and the first posterolateral

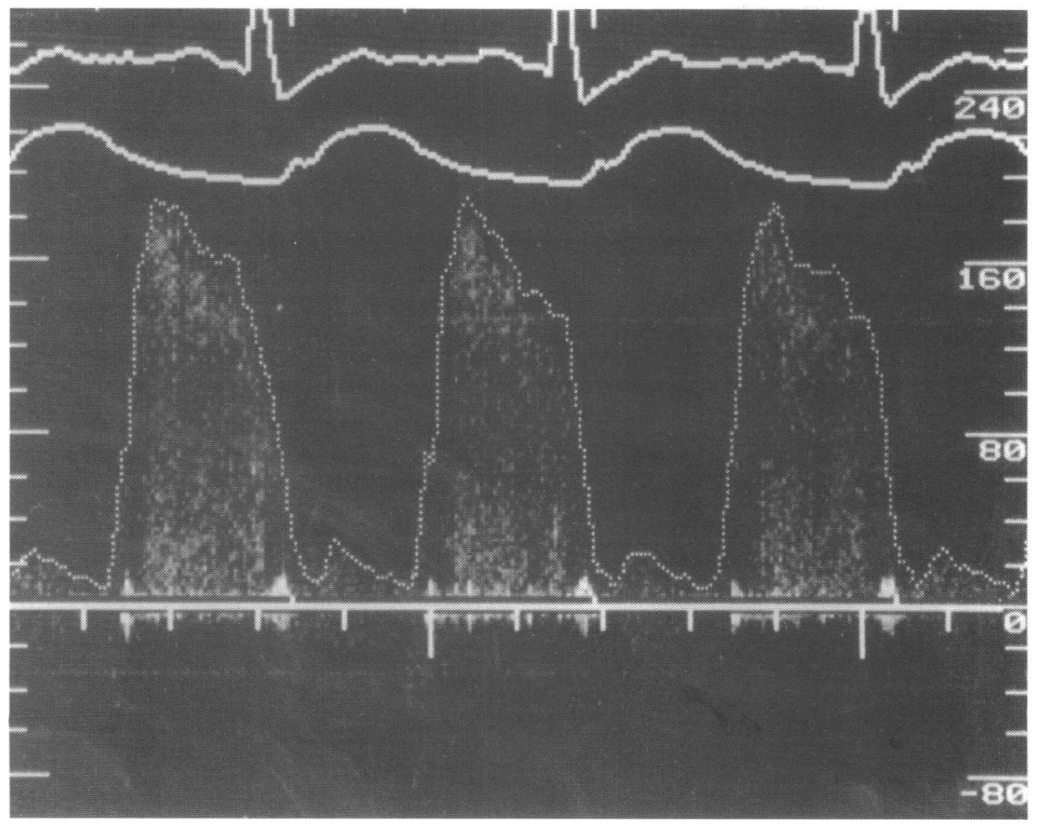

Figure 3 Phasic flow velocity spectrum in the right coronary artery. Top tracing, ECG; Middle tracing, aortic pressure; Bottom tracing, Doppler signal. Vertical axis, flow velocity in $\mathrm{cm} / \mathrm{s}$. Peak flow velocity amounted to $180 \mathrm{~cm} / \mathrm{s}$ and time average of spectral peak velocity to $85 \mathrm{~cm} / \mathrm{s}$ giving a coronary flow rate of $960 \mathrm{ml} / \mathrm{min}$ (vessel diameter $6.8 \mathrm{~mm}$ ). branch of the left circumflex artery appeared tortuous and dilated with multiple fine fistulae to the cavity of the left ventricle (fig 1). The dominant right coronary artery (RCA) also presented a dilated, tortuous morphology with multiple fine communications to the left ventricular cavity via the posterior descending artery (fig 2). Atherosclerotic irregularities with a narrowing of less than $50 \%$ of the vessel diameter were visible in the middle segment of the RCA.

Coronary artery flow velocity was measured with a commercially available Doppler guidewire technique $(0.018$ inch diameter Flowire and FloMap system, Cardiometrics Inc, Mountain View, California, USA) (fig 3). Coronary artery luminal diameters were determined by computer assisted quantitative coronary angiography and amounted to 6.8 and $6.6 \mathrm{~mm}$ in the proximal RCA and LAD, respectively. An estimate of coronary artery flow rate was obtained by the following equation: ${ }^{4}$

$$
\mathrm{Q}_{\mathrm{D}}=\left(\pi \times \mathrm{D}^{2}\right) \times 0.25 \times 0.5 \times \mathrm{APV}
$$

where $Q_{D}=$ Doppler derived time-average flow, $\mathrm{D}=$ vessel diameter, and $\mathrm{APV}=$ time average of the spectral peak velocity. Accordingly, coronary flow rate was calculated as $700 \mathrm{ml} / \mathrm{min}$ and $960 \mathrm{ml} / \mathrm{min}$ in the proximal LAD and RCA, respectively. Measurements of cardiac output with the right ventricle to pulmonary artery thermodilution bolus technique gave an average of $4.81 / \mathrm{min}$.

The patient was discharged on an oral antihypertensive (diltiazem), an antidiabetic (glibenclamide), aspirin, and a cholesterollowering drug (lovastatin). During three years of follow up no further clinical episodes of myocardial ischaemia or heart failure occurred.

\section{Discussion}

Multiple coronary artery-left ventricular fistulae are an uncommon anomaly. According to a recent review, microfistulae originating from all three major coronary arteries and draining in the left ventricle have been reported in only 16 cases. ${ }^{3}$ The pathogenetic origin of the malformation is obscure. Morphological studies suggest a partial persistence of embryonic myocardial sinusoids that arise from endothelial protrusions into the intertrabecular spaces. ${ }^{56}$ Fetal regression of these structures results in the formation of the Thebesian vessels of the adult heart. Thus, interference with developmental changes might produce an abnormally prominent Thebesian system with the morphological appearance of multiple coronary microfistulae.

Most patients in whom this anomaly is diagnosed present with typical or atypical angina pectoris in adult life. The clinical syndrome has been attributed to a coronary steal situation due to the shunting of blood via the low resistance fistulae. ${ }^{7}$ Surprisingly, ischaemic responses during exercise stress testing and thallium scintigraphy were not unanimously observed in the reported cases including the 
present one. In retrospect, the singular appearance of an acute coronary syndrome in our patient most probably resulted from a transitory thrombogenic transformation of the atherosclerotic plaque in the middle segment of the RCA. According to the literature, all patients experienced their first anginal attacks during advanced adulthood (older than 40 years) despite the assumed congenital origin of the malformation. Therefore, the clinical and prognostic relevance of the suspected ischaemic syndrome in multiple coronary artery-left ventricular fistulae appears to be uncertain. ${ }^{5}$

Haemodynamic quantification of shunt flow might provide further insight into the pathophysiology of this anomaly. In the present case, total flow through the left and right epicardial coronary artery was calculated as $1660 \mathrm{ml} / \mathrm{min}$ by using a Doppler guidewire technique. In comparison, total myocardial blood flow in healthy adults has been determined as $150-300 \mathrm{ml} / \mathrm{min}$ at rest ${ }^{8}$ Thus, total left ventricular output might be estimated as the sum of shunt flow $(1400 \mathrm{ml} / \mathrm{min})$ plus systemic flow $(4800 \mathrm{ml} / \mathrm{min})$. Accordingly, leftto-left shunt flow would amount to approximately $23 \%$ of total left ventricular output. It has been speculated that the considerable shunt volume draining into the left ventricle might result in diastolic overload imitating the haemodynamic situation of aortic regurgitation. ${ }^{9}$ In contrast, the estimated shunt fraction of $23 \%$ in our patient cannot be expected to have any negative impact on left ventricular function for it would be classified as being mild even in subjects with aortic regurgitation. Accordingly, no left ventricular enlargement or reduction in ejection fraction were found. The clinical course of the patient was completely uneventful before and after the first anginal episode that led to coronary angiography and diagnosis. In view of these haemodynamic and clinical findings, the question remains whether this entity has any pathophysiological significance or has to be considered as a normal variation due to a prominent Thebesian system. ${ }^{510}$

1 Gillebert C, Van Hoof R, Van de Werf F, De Geest H. Coronary artery fistulas in an adult population. Eur Heart Coronary artery fist

2 Hobbs RE, Millit HD, Raghavan PV, Moodie DS, Sheldon WC. Coronary artery fistulae: a 10-year review. Cleveland Clin $Q$ 1982;49:191-7.

3 Coussement $\mathrm{P}$, De Geest $\mathrm{H}$. Multiple coronary artery-left ventricular communications: an unusual prominent Thebesian system. Acta Cardiologica 1994;49:165-73.

4 Coucette JW, Corl D, Payne HM, Flynn AE, Goto M, Nassi $M$, et al. Validation of a Doppler guide wire for intravascular measurement of coronary artery flow velocity. Circulation 1992;85:1899-911.

5 Cha SD, Maranhao V, Goldberg H. Silent coronary arteryleft ventricular fistula: a disorder of the Thebesian system? Angiology 1978;29:169-73.

6 Black IW, Loo CKC, Allan RM. Multiple coronary arteryleft ventricular fistulae: clinical, angiographic, and patho-
logic findings. Cathet Cardiovasc Diagn 1991;23:133-5.

7 Duckworth F, Mukharji J, Vetrovec GW. Diffuse coronary artery to left ventricular communications: an unusual artery to left ventricular communications: an unusual
cause of demonstrable ischaemia. Cathet Cardiovasc Diagn 1987;13:133-7.

8 Simon R. Coronary circulation. In: Lentner C, ed. Geigy scientific tables, volume 5, heart and circulation. Basel: Ciba Geigy Ltd, 1990:173-81.

9 Reddy K, Gupta M, Hamby RI. Multiple coronary arterioventricular fistulas. Am f Cardiol 1974;33:304-6.

10 Sheikhzadeh A, Stierle U, Langbehn AF, Thoran P, Diederich KW. Generalized coronary arterio-systemic (left ventricular) fistula. $\mathcal{F p n}_{p}$ Heart $\mathcal{f} 1986 ; 27: 533-44$.

\title{
SHORT CASES IN CARDIOLOGY
}

\section{Intractable vasospastic angina}

\author{
C Kurata, A Shimane
}

\footnotetext{
Department of

Medicine III,

Hamamatsu

University School of

Medicine,

Hamamatsu, Japan

C Kurata

A Shimane

Correspondence to:

Dr C Kurata, Department of

Medicine III, Hamamatsu

University School of

Medicine, 3600 Handa-cho,

Hamamatsu 431-31, Japan.

Accepted for publication 29 April 1997
}

A 48 year old woman with drug refractory vasospastic angina had been in our hospital for nine months. Electrocardiograms recorded during angina attacks showed marked elevation of ST segments in the precordial, inferior, and/or lateral leads, often with ventricular arrhythmia or atrioventricular block (fig 1). Coronary angiography revealed no atherosclerotic stenosis, and intracoronary injection of acetylcholine induced total occlusion of the left anterior descending and right coronary arteries despite continuation of high dose calcium antagonists (fig 2).

Calcium antagonists (nifedipine, nisoldipine, amlodipine, benidipine, diltiazem, and verapamil), nitrates (isosorbide dinitrate, isosorbide mononitrate, and nitroglycerin), nicorandil or various combinations of drugs could not prevent her attacks. Blood concentrations of drugs such as diltiazem and nicorandil were much higher than normal therapeutic values. She did not have signs of a generalised vasospastic disorder such as Raynaud's phenomenon nor a family history of coronary artery disease or sudden death. The patient had stopped smoking after admission. Her attacks occurred not only between midnight and early morning but also in the daytime. The trigger of attacks such as emotional distress, exercise, cold, alcohol, or coffee could not be specified. Her regular menstrual cycle with normal variation of blood oestrogen concentrations was not related to the incidence of attacks. 